\title{
(C) OPEN ACCESS \\ Implementing infection prevention practices across European hospitals: an in-depth qualitative assessment
}

\author{
Lauren Clack, ${ }^{1,2}$ Walter Zingg, ${ }^{2}$ Sanjay Saint, ${ }^{3,4}$ Alejandra Casillas, ${ }^{5}$ \\ Sylvie Touveneau, ${ }^{2}$ Fabricio da Liberdade Jantarada, ${ }^{2}$ Ursina Willi, ${ }^{1}$ \\ Tjallie van der Kooi, ${ }^{6}$ Laura J Damschroder, ${ }^{3}$ Jane H Forman, ${ }^{3}$ \\ Molly Harrod, ${ }^{3}$ Sarah Krein, ${ }^{3,4}$ Didier Pittet, ${ }^{2}$ Hugo Sax, ${ }^{1,2}$ PROHIBIT \\ Consortium
}

For numbered affiliations see end of article.

\section{Correspondence to} Professor Hugo Sax, Division of Infectious Diseases and Hospital Epidemiology, University Hospital of Zurich, Raemistrasse 100/HAL14, 8091, Zurich, Switzerland; hugo.sax@usz.ch

LC and WZ contributed equally.

Received 1 December 2017 Revised 8 May 2018 Accepted 13 May 2018 Published Online First 27 June 2018

\section{SLinked}

- http://dx.doi.org/10.1136/ bmjqs-2018-008512

Check for updates

To cite: Clack L, Zingg W, Saint S, et al. BMJ Qual Saf 2018;27:771-780.

\begin{abstract}
Objective The Prevention of Hospital Infections by Intervention and Training (PROHIBIT) project included a cluster-randomised, stepped wedge, controlled study to evaluate multiple strategies to prevent catheterrelated bloodstream infection. We report an in-depth investigation of the main barriers, facilitators and contextual factors relevant to successfully implementing these strategies in European acute care hospitals. Methods Qualitative comparative case study in 6 of the 14 European PROHIBIT hospitals. Data were collected through interviews with key stakeholders and ethnographic observations conducted during 2-day site visits, before and 1 year into the PROHIBIT intervention. Qualitative measures of implementation success included intervention fidelity, adaptation to local context and satisfaction with the intervention programme. Results Three meta-themes emerged related to implementation success: 'implementation agendas', 'resources' and 'boundary-spanning'. Hospitals established unique implementation agendas that, while not always aligned with the project goals, shaped subsequent actions. Successful implementation required having sufficient human and material resources and dedicated change agents who helped make the intervention an institutional priority. The salary provided for a dedicated study nurse was a key facilitator. Personal commitment of influential individuals and boundary spanners helped overcome resource restrictions and intrainstitutional segregation.

Conclusion This qualitative study revealed patterns across cases that were associated with successful implementation. Consideration of the interventioncontext relation was indispensable to understanding the observed outcomes.
\end{abstract}

\section{INTRODUCTION}

Healthcare-associated infection represents the most frequent adverse event affecting intensive care unit (ICU) patients. ${ }^{1}$ Catheter-related bloodstream infections (CRBSIs) affect $6 \%$ of hospitalised patients in Europe. ${ }^{2}$ While CRBSI is amenable to reduction by evidence-based infection control procedures, ${ }^{34}$ variability exists in how hospitals implement infection prevention and control (IPC) practices. $^{5}$

Contributors to variability include differences in organisational context, defined as 'all factors that are not part of a quality improvement intervention itself ${ }^{6}{ }^{6}$ Contextual factors are often cited as playing a role in the success of best practice interventions; however, existing literature reporting on implementation strategies often lacks details about how contextual factors work together and provides limited information about real barriers and facilitators to implementation. ${ }^{78}$ This is of particular interest in the culturally, politically and economically diverse European context. ${ }^{9}$

The current study, termed 'InDepth', was part of the European Commission-funded Prevention of Hospital Infections by Intervention and Training (PROHIBIT) project. The PROHIBIT project aimed, through its multiple work packages, to analyse the variation in implementation of IPC practices across Europe and to test the effectiveness of two interventions of known efficacy on the prevention of CRBSI in European ICUs. ${ }^{10}$ InDepth used a longitudinal qualitative comparative case study to address two questions: (1) what are the main barriers and facilitators to successfully implementing CRBSI prevention procedures?; and 2) what role do contextual factors play?

\section{METHODS}

The methodology has been reported previously. ${ }^{11}$ This in-depth qualitative study 
was conducted in 6 of the 14 hospitals participating in the PROHIBIT randomised controlled study on CRBSI prevention. ${ }^{12}$ After a 6 -month baseline period, three hospitals were computer-randomised every quarter to one of three interventions: (1) hand hygiene strategy based on World Health Organization (WHO) recommendations ${ }^{13} ;(2)$ comprehensive central venous catheter (CVC) insertion strategy ${ }^{14}$; and (3) both interventions combined. Study nurses and ICU physicians from participating hospitals were invited to a two-day workshop where they received training regarding study implementation by the PROHIBIT study team.

\section{Methods}

Here, we report our methods according to the Consolidated Criteria for Reporting Qualitative Research. ${ }^{15}$ We purposefully selected InDepth hospitals from PROHIBIT participants by 'extremes' and 'intensity', that is, those most likely to succeed or to be challenged with the implementation and therefore richly demonstrate potential barriers and facilitators. ${ }^{16-18}$ Selection was based on a questionnaire and a telephone interview with the on-site investigators. Two-day site visits were conducted prior to and 1 year into the intervention of the PROHIBIT study on CRBSI prevention. Participants were purposefully selected for semistructured interviews at multiple hierarchy levels and from key departments including hospital administration, IPC professionals and ICU teams. ${ }^{16}{ }^{17}$ Between visits, telephone interviews were conducted with the PROHIBIT on-site investigators. ${ }^{16}$ All interviews were audio-recorded and transcribed verbatim, and transcripts were reviewed for correctness. Direct, non-participatory observations of daily routines in all ICUs were conducted during each site visit, and detailed notes were included in the analysis. ${ }^{19}$ This research was guided a priori by several theories and implementation frameworks that informed our data collection (eg, semistructured interview guide) and analyses (eg, coding framework and site summaries). Namely, our conceptual model followed a temporal scheme based on an adapted 'Diffusion of Innovation' framework, and our analysis was guided by the distinction between domains made within the Consolidated Framework for Implementation Research. These domains included the intervention itself, the inner (hospital and ICU) and outer (country and region) settings, the involved individuals and the implementation process. ${ }^{20-24} \mathrm{We}$ analysed the data by establishing structured individual site summaries that incorporated data from both site visits. Themes were identified using both inductive (first site visit) and deductive (second site visit) approaches. For cross-case analysis, we used a stacking technique, whereby cases were grouped according to common traits into matrices and differences, and similarities were investigated across the stacked cases. Information power saturation was achieved by focusing on three meta-themes for the second site visit by having a combination of highly specific stakeholders for the study questions and by applying a strong interview dialogue when interviewing most of the stakeholders during both site visits. ${ }^{25}$ We remained blinded to quantitative results of the CRBSI prevention study during data collection and until the final stages of qualitative analysis. Our research team consisted of two female social scientists (LC and UW), three physicians (one female) with training in infectious diseases and IPC (WZ, AC and HS), a female IPC nurse (ST), and a male administrative manager (FdLJ), who were all employed as clinicians and researchers at the University Hospital of Geneva and the University Hospital of Zurich at the time of the study. Consultation on qualitative research was provided by researchers with extensive experience in the field (SS, LJD, JHF, MH and SK).

\section{Measures of implementation success}

We developed a three-part qualitative definition of implementation success based on the literature (box 1). ${ }^{26}$ Ratings of 'low', 'middle' or 'high' for each of the three qualitative measures of implementation were assigned based on researcher consensus. Quantitative outcomes were obtained from the PROHIBIT randomised controlled study. ${ }^{12}$ These were transformed into semiquantitative categories using thresholds based on terciles of overall compliance and reported CRBSI incidence densities. ${ }^{27-29}$

\section{Ethics}

Participation was voluntary. All interviewees provided written informed consent.

\section{RESULTS}

\section{Data collected}

A total of 129 interviews (about 133 hours) were conducted, with an average of 11 (8-13) interviews per site visit. Interviewees represented hospital administration, IPC and ICU leadership and staff (box 2). We conducted 12 additional phone interviews with

\section{Box 1 Qualitative definition of implementation} success

1. Satisfaction with the intervention programme, also referred to as 'acceptability', ${ }^{26}$ was based on interviewees' experience with the programme design, the implementation process and the outcome.

2. Extent of 'intervention fidelity', or the extent to which the intervention was implemented as specified by the PROHIBIT study protocol ${ }^{2326}$ was assessed by interviews and triangulated based on field notes.

3. Resolution of intervention-to-context fit, that is, adaptation, local re-engineering and innovation, demonstrating that the programme was adapted to the local context to assist implementation ${ }^{41}$ and sustainability. ${ }^{42}$ 


\section{Box 2 InDepth study interviewees}

\section{Top management level $(n=26)$ : \\ - Chief executive officer \\ - Director of nursing \\ - Medical director}

\section{Infection prevention and control (IPC) programme* $(n=41)$ : \\ - Head of IPC \\ - IPC practitioners or nurses \\ - IPC physicians \\ - Epidemiologists}

\section{Intensive care unit (ICU) leaders $(n=21)$ : \\ - ICU head physician \\ - ICU head nurse}

\section{ICU frontline workers $(n=41)$ :}

- ICU nurses

- ICU link nurse for IPC

- ICU physicians or anaesthesiologists

*The Prevention of Hospital Infections by Intervention and Training (PROHIBIT) on-site investigator and PROHIBIT study nurse, based in the infection prevention and control programme, were interviewed in all hospitals.

the on-site investigators, before and after site visits. Furthermore, 41 hours of direct observation were conducted.

\section{Overall implementation success}

As reflected in table 1, implementation outcomes revealed complex patterns reflecting diverse local contexts rather than distinct barriers and facilitators. To summarise the qualitative measures of implementation success, we report our findings as three metathemes: 'implementation agendas', 'resources' and 'boundary-spanning'. Regarding the quantitative outcomes, hospitals with relatively high baseline CRBSI rates achieved impressive reductions. ${ }^{12}$ The reduction in hospitals with already low baseline CRBSI rates was not significant. All but two hospitals increased overall hand hygiene performance.

\section{Implementation agendas}

Each hospital established its unique implementation agenda, which did not always align with the intended goal of PROHIBIT but affected various aspects of implementation success. Hospitals consistently cited PROHIBIT's funding for a 0.5 full-time equivalent (FTE) nurse as an attractive incentive to participate; however, deployment varied according to local context and goals.

\section{Misaligned implementation agendas}

Hospitals in countries with higher healthcare expenditure and randomised to a PROHIBIT study arm for which they had already invested in the past intended to use the 0.5 FTE nurse to promote IPC activities other than specified by the PROHIBIT protocol. For example, in a hospital with an extensive history of hand hygiene promotion that was randomised to the hand hygiene arm, the IPC head physician expressed that they had actually hoped to be randomised to the CVC study arm. IPC staff in this hospital consequently decided to use the PROHIBIT-funded 0.5 FTE nurse to increase the presence of IPC in the ICU beyond hand hygiene, for example, eliminating the use of long-sleeve apparel. The head ICU physician in a hospital randomised to the CVC intervention arm shared a similar experience.

We already used many of the recommended routines [...]. The reason for me to participate in this programme was basically that I simply wanted to know what the deal is in my department ... The improvements that came out of it were never my primary focus [...]. We don't routinely collect data about catheter infections [...]. Had we not participated I wouldn't know anything about it. (Head ICU physician)

Hospitals that were randomised to study arms for which they did not perceive a need for further improvement subsequently established implementation agendas that misaligned with formal PROHIBIT core contents, yet they still considered PROHIBIT a success.

Aligned implementation agendas

Hospitals whose needs aligned more closely with the goals of PROHIBIT, particularly hospitals with high baseline CRBSI rates and located in countries with lower healthcare expenditures, used PROHIBIT participation and the 0.5 FTE nurse to standardise procedures and reduce infection rates.

I was really hoping for good results from this project because I saw it as offering a unified [CVC insertion] procedure for staff members. They are coming from different generations with different types of education and there is no unified procedure. (Head ICU nurse)

For me it was the occasion to internalise something more in the field of catheter infection prevention and to establish a collaboration outside of the hospital. And [...], since the experience with the implementation of the former protocol was not easy, I appreciated to have some support in that sense. (Nursing director)

I think we really expected to drop CVC rates and it happened, and we expected to improve our hand hygiene compliance and it happened. (IPC head physician)

Such hospitals, whose implementation agendas aligned well with PROHIBIT project objectives, achieved high levels of satisfaction with the implementation as well as implementation fidelity (table 1). 


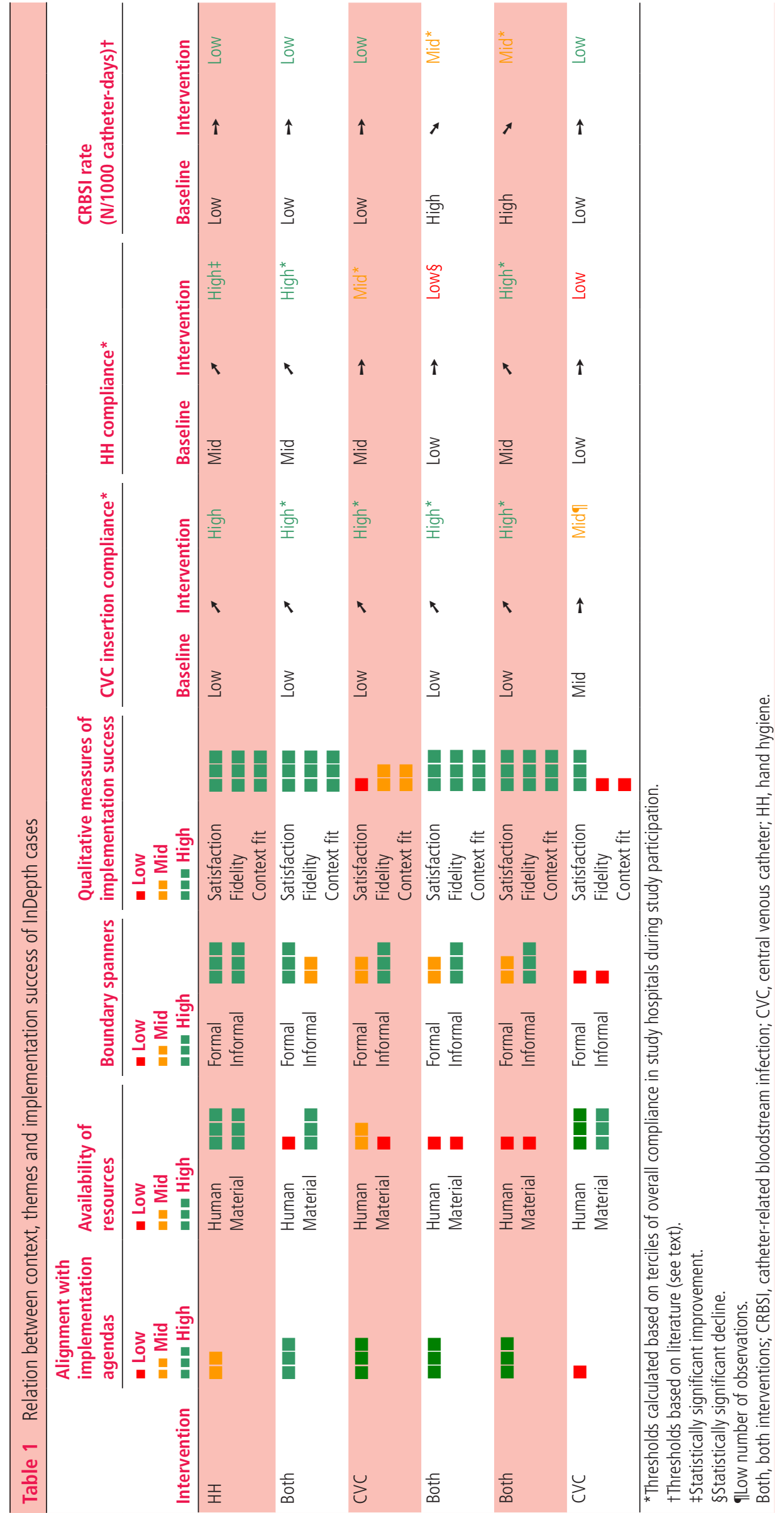




\section{Resources}

Limited resources-both material and human-were mentioned in all hospitals, although the level of impact was different. The distribution of limited resources reflected the organisation's relative priorities, sometimes shifting over time, particularly driven by change agents and disruptive events.

Human resources in countries with higher health expenditures In countries with higher health expenditures, the 2008 economic crisis led to governmental limits on hiring. Healthcare professionals leaving the hospital were not replaced, and the performance of highly specialised units such as the ICU suffered. Having the 0.5 FTE PROHIBIT-funded nurse was specifically mentioned as a facilitator.

From an engagement point of view, [having the PROHIBIT nurse] has been very positive. [She] developed great relationships with the staff up there [in the ICU] [...] and we wouldn't have that luxury in other areas because there's [only] so many staff. (IPC head nurse)

PROHIBIT-funded staff increased satisfaction and helped intervention fidelity.

Human resources in countries with lower health expenditures

The economic crisis at the time of the study particularly affected hospitals in countries with lower healthcare expenditures, resulting in dramatic salary reductions. As a result, nurses and doctors from these hospitals had multiple jobs, continued to work beyond retirement age or left the country. One nurse shared that 'almost everybody' in the hospital had multiple jobs. Alternatively, many nurses worked overtime.

Because wages are very low and the work is very tough... they try to find jobs where they might not earn more but the working hours are better. Like when these [name] supermarkets opened then a lot of nurses went there to work. (Hospital CEO)

Difficult working conditions (eg, 24-hour shifts, high workload, having multiple jobs and low salaries) that threatened individual motivation were described as a barrier to PROHIBIT.

But a nurse has five patients or at night has eight, she might be so tired. Of course, they don't really care about this [PROHIBIT project]. And they have low salaries and are thinking about going abroad. (IPC head physician)

Emigration to Western European countries after joining the European Union was a direct barrier to implementation in Eastern European hospitals, particularly concerning trained staff.

For the last twoyears, due to European education programmes...there are a lot of European universities and countries coming here to recruit our nurses to go to work abroad...Great Britain, Germany, England, and Norway. (Nursing director)

Some time ago, this hospital, you couldn't get a place to work here...it was an honour to work in this hospital. Nowadays, you can't find an ICU head physician. You can't find a head nurse for the ICU... because the other hospitals pay them more. It's very messed up. (IPC head physician)

In another Eastern European hospital, emigration resulted in significant ICU physician turnover.

We don't have [physicians age 35-50] anymore because they went to England, they went to Germany, to earn a bit of money, to start a family, I mean a feasible life I guess. (ICU physician)

Such rapid staff turnover was mentioned as a barrier to implementation in several hospitals and directly impacted on PROHIBIT because it was challenging to keep new staff trained in PROHIBT protocols. PROHIBIT implementation was also specifically challenged when staff turnover affected the study nurses responsible for PROHIBIT implementation. Yet, hospitals that succeeded in making PROHIBIT a priority despite limited human resources perceived the programme as a success.

\section{Material resources}

Lack of material resources was one of the main challenges to maintaining implementation fidelity. Some hospitals failed to procure alcohol-based chlorhexidine skin antiseptic, large sterile drapes, insertion kits and 'line' carts. In an extreme case, alcohol-based handrub often ran out, competing in the same budget allocation with antibiotics. In this hospital, it emerged that nurses were trying to reduce consumption of alcohol-based handrub and personal protective equipment (eg, gloves) to cut costs.

The budget we get for infection control is included in the budget for the medicine that one of the units get...if a medicine is needed, then of course, that will get priority in front of the disinfectants. (IPC head physician)

Because they are saving on the handrub. The nurses think that this is the only thing they can save on. (IPC head physician)

In another hospital, a perceived cost barrier prevented the adoption of a large sterile drape.

Because I'm not convinced completely that this would make this huge difference in quality, we didn't introduce the large drape until now. (Hospital CEO)

Non-availability on the national market, in the case of alcohol-based 2\% chlorhexidine skin antiseptic, occurred in more than one country. Local adaptation, a qualitative measure of implementation success, was achieved in sites that locally produced insertion kits and appropriate skin antiseptics. 
It was prohibited to buy the whole [insertion kit] so we had to pack it ourselves here. (IPC head physician)

In one case, procurement was only possible because of the informal connections the ICU head physician had with influential people in procurement.

\section{Distribution of resources}

The distribution of resources towards or away from the PROHIBIT project played an important role in implementation success. Change agents, both local and external to the hospital, as well as disruptive events were critical in shaping resource distribution, as discussed in the following sections.

\section{Local change agents}

Hospitals succeeded in PROHIBIT implementation despite resource limitations through the actions of local change agents. In a hospital with low salaries where staff had multiple jobs, the ICU head physician, a local change agent, negotiated with the hospital director to secure higher wages for ICU physicians in hopes that they would work exclusively in that ICU, which removed a potential barrier to PROHIBIT implementation.

My physicians are paid quite well, I suppose much more than others in the hospital, because when I started work here, I needed 16 people, and I had only eight physicians. So I proposed to pay these eight and they work in one place, a lot of hours, [but] less than the past. So they earn quite good money. It's difficult. Because in [this country] doctors work in one hospital, and next go to work in another practice, or another hospital. And I insist my physicians work only here.

(ICU head physician)

This example demonstrates how a local change agent overcame resource limitations through negotiations to ensure appropriate remuneration. In contrast, missing change agents or lack of local leadership was a significant barrier. In one hospital, the nurse and the physician who received PROHIBIT training left the hospital shortly after PROHIBIT began and their responsibilities were not reassigned. Furthermore, the on-site investigator delegated part of the responsibilities to a colleague. This hospital failed to allocate the human resources necessary to support the PROHIBIT intervention.

\section{External change agents}

In some sites, PROHIBIT was an external driving force, unlocking local resources that would not have been otherwise available. Multiple IPC head physicians mentioned that having a specific project mandate facilitated the implementation.

It's sometimes easier to get something introduced if there's a specific reason it has to be done or if you have a national mandate for it. (IPC head physician)
For us PROHIBIT makes for very good work because I have a new argument. We have a programme. You have to! (IPC head physician)

By establishing the intervention within a fixed time and place, PROHIBIT provided stimulation to motivate staff and an argument to concentrate resources around the prevention of CRBSI.

\section{Disruptive events}

Disruptive events, defined here as circumstances that interrupt the routine functioning of an organisation, can have a profound impact-either positive or negative-on the distribution of resources. For example, an outbreak in one hospital increased awareness of the importance of IPC and helped to generate resources.

We had an outbreak of VRE (vancomycinresistant enterococcus) back a couple of months ago... everybody got together and said look, what can we do to improve? [And we] did a big overhaul. (ICU nurse)

However, relocating an entire hospital—as happened at another site shortly after enrolling in PROHIBITshifted the focus away from IPC. The relocation of the hospital represented a major barrier to data collection and implementation of the CRBSI prevention strategy.

We suspended hand hygiene observations for threemonths because everyone was busy with the move. (IPC nurse)

This PROHIBIT project became a little less important and moved to the second level of importance, of priority. (ICU nurse)

\section{Boundary spanning}

We define boundary spanners as individuals who have multiple roles, traverse institutional boundaries and accelerate change. ${ }^{20}$ Communication in participating hospitals varied particularly in relation to whether the hospital had a culture of formal or informal networks based on interpersonal relationships. In either, boundary-spanning individuals with positive attitudes towards the project were facilitators to implementation. Through their strong ties in multiple areas of an organisation, such individuals are able to influence their colleagues and facilitate implementation.

Boundary spanning across formal structures

We saw boundary spanners who were influential through formal networks both in vertical and horizontal hierarchies. We identified two individuals who acted as boundary spanners within the formal structures of an institution that was otherwise described as having a top-down culture. They had joint appointments in the ICU and IPC programme, allowing them to more effectively influence their colleagues to follow PROHIBIT procedures. 
When [IPC] wants to implement something, I usually do it... because it's easier for me to say, I know the equipment, I know the staff, I know how it's done, and I know the ICU. (ICU/IPC nurse)

Another boundary-spanning physician, who was previously appointed as medical director, used connections to the hospital CEO to purchase the necessary materials for PROHIBIT. Importantly, the influence of boundary-spanning individuals was further enhanced by the fact that they were described by their colleagues as well respected and having 'huge knowledge about both intensive care and microbiology or epidemiology'.

Another example of boundary spanning was observed in a hospital that was described by the IPC head physician as not having a 'big hierarchy'. Boundary spanning occurred across formal structures, where individuals from different wards came into contact through interdisciplinary committees on a regular basis. Such committees were designed to support the development of best practices across hospitals in the same network.

Because there is a very good structure. We have all of the committees... patient safety, the risk advisory committee, finance, all of the health and safety. [...] [For] example, this morning at our patient safety committee meeting, we had specialised surgeons, emergency medicine physicians, as well as patient safety. And we had a report that was circulated in relation to our hand hygiene. [...] So I got my opportunity at that meeting to say, "the medics have pulled us down a bit this month, what are we going to do about that?' (Nursing director)

We're trying to have some infection control committee reports with the other two infection control committees from the other hospitals, reporting into the steering committee. [...] It's good when you have the CEOs of the other hospitals, to try and get a path across. You know, some may come up with very good things we can think about [ourselves]. It's nice just to share. (IPC physician)

According to this nursing director, such committees were important for internal networking and they appeared to be important to staff satisfaction, providing a forum for staff to feel empowered.

\section{Boundary spanning across informal networks}

We also saw boundary spanning via informal networks, such as friendships among clinicians.

I have a lot of people above me that decide, but the people that I consider my real leader/chief are directly connected with me and are friends of mine. So it's quite easy for me to work with them. We go to the restaurant. (ICU physician)

In this hospital, effective boundary spanners were those who took advantage of personal relationships. For example, the previous head of IPC had established informal links with hospital administration. $\mathrm{He}$ used these links to put IPC high on the list of institutional priorities and convince the CEO to participate in PROHIBIT. When this individual left the hospital, however, it was a challenge to maintain the priority that had previously been attributed to the project.

Before, [the IPC department] felt that we were respected and heard, but now, we don't feel that we are heard anymore. ...[T] leadership and everyday work in the hospital has drifted really apart. (IPC nurse)

\section{Lack of boundary spanning}

In some hospitals, the lack of boundary spanners was an obvious barrier, particularly when hierarchical structure and institutional silos slowed down the pace of progress.

There's a very strong hierarchy here in [this country] and the hierarchy is so respected and there's no way I would go to the nurse director and ask for something, but I would go to the nurse superior, and the superior to his superior and so on... Everything's too slow. Things are not moving forward. You have to ask 10 people if this is ok. (ICU head nurse)

If you want to change things ... there are so many different bodies to be asked ... it's a little bit like Kafka. (IPC physician)

Implementing a project requiring the collaboration of several individual departments was difficult in this hospital. We have learnt from IPC professionals that once PROHIBT was introduced, the ICUs were left to continue as they saw fit.

I am not really so close to these kinds of wards ... I never heard anything about it, so it's working. I did not hear something from my people so I presume that it is working. (IPC physician)

I presented the PROHIBIT study per se ... It's maybe too much because they know, they are professors, I mean they know what they are doing, they know how to insert the catheters. (IPC nurse)

The IPC nurse was not aware of training or promotional activities that had taken place in the ward, and it was later confirmed from an ICU physician that there was no education or feedback on compliance. We suspect that the IPC nurse responsible for training did not feel empowered to provide training to ICU staff. This may be due to the existing organisational culture of departments, functioning as independent structures and being led by independent leaders. The ICU staff in this hospital did not feel involved in the programme and did not promote the intervention.

\section{Interactions between context and intervention}

Three principal recurring scenarios emerged relating to the three main themes: 'implementation agendas', 'resources', and 'boundary-spanning'. First, a scenario where hospitals with limited previous IPC quality 
improvement experience, relatively high baseline CRBSI rates and limited financial and human resources benefited optimally from the quality improvement initiative, as exemplified by the second, fourth and fifth hospitals in table 1 . Here, attention and focus attributed to PROHIBIT were leveraged by boundary spanning individuals to overcome the limited resources available and significantly curb infection rates. External change agents, specifically, the source of the intervention and connection to an international network, contributed to establishing the necessary institutional focus in this context. In a second scenario, disruptive events drew attention away from the project in a context that would have otherwise been favourable, as seen withthe third hospital in table 1 . This second scenario exemplifies contextual influences that are outside the control of stakeholders. In such a scenario, the typical life cycle of an intervention may be too short; postponing or prolongation may be beneficial. Finally, a third scenario, set in the context of higher healthcare expenditure and markedly low baseline CRBSI rates, where PROHIBIT resources helped to promote IPC activities outside the scope of the initiative, as in the first and sixth hospitals in table 1 . While in this scenario participants were unable to further lower CRBSI rates or significantly improve quantitative process indicators, they were satisfied with the intervention, primarily thanks to improvements peripheral to PROHIBIT.

\section{DISCUSSION}

Our multicentre qualitative study provides several key insights for implementing quality improvement initiatives in the culturally diverse European context. First, hospitals developed 'implementation agendas', which are context driven and may or may not align with formal project goals. These agendas shaped what followed, including participants' perception of success or failure. Second, successful implementation gravitated around the ability of the institution to generate focus on the programme. The salary that came with PROHIBIT for a 0.5 FTE dedicated study nurse was a key facilitator. In some cases, this nurse also became an internal change agent. Finally, personal commitment of influential individuals, especially when in a boundary-spanning position, helped overcome resource restrictions and intrainstitutional segregation. A key to success was finding influential boundary-spanning individuals with positive attitudes towards the programme to traverse institutional limits and accelerate change. Such success, however, depends on the continued presence and active engagement of these individuals.

Similar to other qualitative inquiries, ${ }^{7}{ }^{30}$ we found that the context-intervention relation was key to understanding the observed study outcomes. In order to advance the science of quality improvement, multifaceted interventions need to report on context in addition to effectiveness. ${ }^{31}$ Without information on the context in which implementation took place, interpretation and generalisation of the results are limited. The outcome of a change initiative is a function of shared commitment to the change among members of an organisation as well as confidence in their collective ability to implement the change. ${ }^{32}$ Individual change agents are essential in catalysing change within the social architecture of an organisation. ${ }^{7}$ With some notable exceptions, contextual factors are poorly reported in the literature. ${ }^{33}$ Specifically related to PROHIBIT's focus on reducing CRBSI rates, among the 79 studies on effectiveness of insertion and maintenance bundles to prevent CRBSI in critically ill patients in a recent meta-analysis, ${ }^{34}$ detailed contextual information was available only from two studies. ${ }^{35} 36$ Mixed-method studies such as ours, ${ }^{12}$ and others, ${ }^{37}$ offer the most complete information on how to adopt, adapt and replicate a quality improvement intervention.

Dixon-Woods et al conducted a qualitative assessment ${ }^{38}$ of the 'Matching Michigan' project to reduce ICU-acquired CRBSI in England, ${ }^{36}$ based on an originally successful intervention conducted in Michigan, USA. ${ }^{35}$ The failure to replicate the study was explained by dissimilarities of participating sites' outer and inner contexts. English hospitals perceived the intervention as a top-down prescription due to previous experiences with state-led initiatives. PROHIBIT, however, was not necessarily perceived as a top-down prescription by participants. In fact, being led by a central unit outside of the participants' countries was a facilitator, particularly in Eastern Europe. The effect of the original intervention in Michigan has also been analysed using qualitative methods. ${ }^{39}$ This study differed from ours as it was retrospective and collected data from the study team and not from participating hospitals. The results, however, were similar regarding the importance of belonging to a network with peer interaction and extensive room for local adaptation. Our findings also add that the source of the intervention, its imprimatur, matters according to country context-being especially important in Eastern Europe.

This study's major strength is that it provides context to implementing CRBSI prevention practices as part of a multicentre and multinational trial. The diverse backgrounds of participating sites provided the necessary data to study the influence of various settings on the capacity of a single hospital to implement a quality improvement intervention. Moreover, it provides results stemming from the reality of a diverse European context and informs the design of future quality improvement initiatives. Furthermore, the longitudinal assessment, with its corresponding evaluations before and during the intervention, allowed us to monitor the evolution of the implementation experience and assess related and unrelated changes. Finally, rich data collection and prolonged time in the field of a multidisciplinary research group provided a platform for effective triangulation. 
Our study should be considered in light of some limitations. First, due to the the sampling strategy of PROHIBIT - which only accepted larger hospitals with established IPC programmes - our findings may not apply to smaller hospitals or those lacking access to IPC knowledge. Second, the limited length of our visits may have prevented the observation of subtle attitudes that may have been uncovered through longer ethnographic evaluation. Additionally, sustainability of the achieved improvements cannot be truly judged from the present data. A follow-up study assessing the sustainability of such quality improvement programmes is warranted, particularly to evaluate project evolution once funding for the 0.5 FTE study nurse ceases. Finally, although these qualitative results may not be simply extrapolated to all other European hospitals, important insights may be drawn by hospitals wishing to implement IPC practices and for the design of future multinational quality improvement programmes. ${ }^{40}$

In conclusion, hospital-specific implementation goals, often diverging from the formal goals of PROHIBIT, largely governed the subsequent focus of the programme. In a context of omnipresent resource constraints, the ability to create focus around and provide the necessary resources for these programmes was important. This was most often achieved through the personal commitment of boundary spanners who helped overcome resource restrictions and intra-institutional segregation. Participation in a multinational programme provided accountability and facilitation.

\footnotetext{
Author affiliations

'Division of Infectious Diseases and Hospital Epidemiology, University of Zurich, University Hospital of Zurich, Zurich, Switzerland

${ }^{2}$ Infection Control Programme, University of Geneva Hospitals, Geneva, Switzerland

${ }^{3}$ Center for Clinical Management Research, VA Ann Arbor Healthcare System, Ann Arbor, Michigan, USA

${ }^{4}$ Department of Internal Medicine, University of Michigan Health System, Ann Arbor, Michigan, USA

${ }^{5}$ Division of General Internal Medicine and Health Services Research, Department of Medicine, UCLA David Geffen School of Medicine, Los Angeles, California, USA

${ }^{6}$ Centre for Infectious Disease Control, National Institute for Public Health and the Environment, Bilthoven, The Netherlands
}

Collaborators PROHIBIT Consortium: R Ahmad, B Allegranzi, B Cookson, M Dettenkofer, P Gastmeier, H Grundmann, S Hansen, P B Heczko, A Holmes, Y Kyratsis, A Magiorakos, M Martin, D Pittet, H Richet, H Sax, E Szilágyi, B van Benthem, $\mathrm{T}$ van der Kooi, A W Wu, and W Zingg.

Contributors LC, WZ, AC, ST, FdLJ and HS were involved in study design, data collection and analysis. SS, LJD, JHF, MH and SK were involved in study design and provided expert guidance in qualitative research methods. UW contributed to qualitative analysis. TvdK served as a liaison between the InDepth study group and the quantitative work package. DP obtained project funding and was involved in study design. LC and HS drafted the initial manuscript, which was revised by all coauthors. LC, WZ, DP and HS are guarantors.
Funding The study was funded by FP7 Health (241928) and The Swiss National Science Foundation (grant no. 32003B_149474)

Competing interests None declared.

Patient consent Not required.

Ethics approval The study protocol was approved by the Institutional Review Board at participating hospitals.

Provenance and peer review Not commissioned; externally peer reviewed.

Open access This is an open access article distributed in accordance with the Creative Commons Attribution Non Commercial (CC BY-NC 4.0) license, which permits others to distribute, remix, adapt, build upon this work noncommercially, and license their derivative works on different terms, provided the original work is properly cited and the use is non-commercial. See: http://creativecommons.org/licenses/ by-nc/4.0

(C) Article author(s) (or their employer(s) unless otherwise stated in the text of the article) 2018. All rights reserved. No commercial use is permitted unless otherwise expressly granted.

\section{REFERENCES}

1 Vincent JL, Rello J, Marshall J, et al. International study of the prevalence and outcomes of infection in intensive care units. JAMA 2009;302:2323-9.

2 Zarb P, Coignard B, Griskeviciene J, et al. The European Centre for Disease Prevention and Control (ECDC) pilot point prevalence survey of healthcare-associated infections and antimicrobial use. Euro Surveill 2012;17.

3 Zingg W, Holmes A, Dettenkofer M, et al. Hospital organisation, management, and structure for prevention of health-care-associated infection: a systematic review and expert consensus. Lancet Infect Dis 2015;15:212-24.

4 Storr J, Twyman A, Zingg W, et al. Core components for effective infection prevention and control programmes: new WHO evidence-based recommendations. Antimicrob Resist Infect Control 2017;6:6:6.

5 Saint S, Kowalski CP, Forman J, et al. A multicenter qualitative study on preventing hospital-acquired urinary tract infection in US hospitals. Infect Control Hosp Epidemiol 2008;29:333-41.

6 Øvretveit J. Understanding the conditions for improvement: research to discover which context influences affect improvement success. BMJ Qual Saf 2011;20 Suppl 1(Suppl 1):i18-i23.

7 Krein SL, Damschroder LJ, Kowalski CP, et al. The influence of organizational context on quality improvement and patient safety efforts in infection prevention: a multi-center qualitative study. Soc Sci Med 2010;71:1692-701.

8 Grimshaw JM, Thomas RE, MacLennan G, et al. Effectiveness and efficiency of guideline dissemination and implementation strategies. Health Technol Assess 2004;8-1-72.

9 House R, Hanges P, Javidan M, et al; Culture, Leadership, and Organizations. Thousand Oaks, CA, USA: Sage Publications, 2004.

10. Prevention of Hospital Infections by Intervention and Training. https://plone.unige.ch/prohibit (accessed 26 Jun 2015).

11 Sax H, Clack L, Touveneau S, et al. Implementation of infection control best practice in intensive care units throughout Europe: a mixed-method evaluation study. Implement Sci 2013;8:24.

12 van der Kooi T, Sax H, Pittet D, et al. Prevention of hospital infections by intervention and training (PROHIBIT): results of a pan-European cluster-randomized multicentre study to 
reduce central venous catheter-related bloodstream infections. Intensive Care Med 2018;44:48-60.

13. World Health Organization. WHO guidelines on hand hygiene in health care. Geneva, Switzerland: World Health Organization, 2009. http://apps.who.int/iris/bitstream/10665/ 44102/1/9789241597906_eng.pdf

14 Zingg W, Cartier V, Inan C, et al. Hospital-wide multidisciplinary, multimodal intervention programme to reduce central venous catheter-associated bloodstream infection. PLoS One 2014;9:e93898.

15 Tong A, Sainsbury P, Craig J. Consolidated criteria for reporting qualitative research (COREQ): a 32-item checklist for interviews and focus groups. Int J Qual Health Care 2007;19:349-57.

16 Patton MQ. Qualitative research and evaluation methods. 3 ed. Thousand Oaks: Sage Publications, 2002.

17 Kuper A, Lingard L, Levinson W. Critically appraising qualitative research. BMJ 2008;337:a1035.

18 Malterud K, standards Qualitative research:. challenges, and guidelines. Lancet 2001;358:483-8.

19 Strauss AL, Corbin JM. Basics of qualitative research. Thousand Oaks, CA, USA: Sage Publications, Inc, 1998.

20 Greenhalgh T, Robert G, Macfarlane F, et al. Diffusion of innovations in service organizations: systematic review and recommendations. Milbank Q 2004;82:581-629.

21 Weick KE, Sutcliffe KM, Obstfeld D. Organizing and the Process of Sensemaking. Organization Science 2005;16:409-21.

22. Greenwood R, Oliver C, Suddaby R, et al. The SAGE Handbook of Organizational Institutionalism. Thousand Oaks, CA, USA: Sage Publications, Inc, 2008.

23 Damschroder LJ, Aron DC, Keith RE, et al. Fostering implementation of health services research findings into practice: a consolidated framework for advancing implementation science. Implement Sci 2009;4:50.

24 Kitson A, Harvey G, McCormack B. Enabling the implementation of evidence based practice: a conceptual framework. Qual Health Care 1998;7:149-58.

25 Malterud K, Siersma VD, Guassora AD. Sample Size in Qualitative Interview Studies: Guided by Information Power. Qual Health Res 2015.

26 Proctor E, Silmere H, Raghavan R, et al. Outcomes for implementation research: conceptual distinctions, measurement challenges, and research agenda. Adm Policy Ment Health 2011;38:65-76.

27 Dudeck MA, Edwards JR, Allen-Bridson K, et al. National Healthcare Safety Network report, data summary for 2013, Device-associated Module. Am J Infect Control 2015;43:206-21.
28 Rosenthal VD, Maki DG, Mehta Y, et al. International Nosocomial Infection Control Consortium (INICC) report, data summary of 43 countries for 2007-2012. Deviceassociated module. Am J Infect Control 2014;42:942-56.

29 Wetzker W, Bunte-Schönberger K, Walter J, et al. Compliance with hand hygiene: reference data from the national hand hygiene campaign in Germany. J Hosp Infect 2016;92:328-31.

30 McCullough MB, Chou AF, Solomon JL, et al. The interplay of contextual elements in implementation: an ethnographic case study. BMC Health Serv Res 2015;15:62.

31 Shekelle PG, Pronovost PJ, Wachter RM, et al. Advancing the science of patient safety. Ann Intern Med 2011;154:693-6.

32 Weiner BJ. A theory of organizational readiness for change. Implement Sci 2009;4:67.

33 Kringos DS, Sunol R, Wagner C, et al. The influence of context on the effectiveness of hospital quality improvement strategies: a review of systematic reviews. BMC Health Serv Res $2015 ; 15: 277$.

34 Ista E, van der Hoven B, Kornelisse RF, et al. Effectiveness of insertion and maintenance bundles to prevent central-lineassociated bloodstream infections in critically ill patients of all ages: a systematic review and meta-analysis. Lancet Infect Dis 2016;16:724-34.

35 Pronovost P, Needham D, Berenholtz S, et al. An intervention to decrease catheter-related bloodstream infections in the ICU. N Engl J Med 2006;355:2725-32.

36 Bion J, Richardson A, Hibbert P, et al. 'Matching Michigan': a 2-year stepped interventional programme to minimise central venous catheter-blood stream infections in intensive care units in England. BMJ Qual Saf 2013;22:110-23.

37 Benning A, Ghaleb M, Suokas A, et al. Large scale organisational intervention to improve patient safety in four UK hospitals: mixed method evaluation. $B M J$ 2011;342:d195.

38 Dixon-Woods M, Leslie M, Tarrant C, et al. Explaining Matching Michigan: an ethnographic study of a patient safety program. Implement Sci 2013;8:70.

39 Dixon-Woods M, Bosk CL, Aveling EL, et al. Explaining Michigan: developing an ex post theory of a quality improvement program. Milbank Q 2011;89:167-205.

40 Malterud K. The art and science of clinical knowledge: evidence beyond measures and numbers. Lancet 2001;358:397-400.

41. Rogers EM. Diffusion of innovations. 5 ed. New york, NY, USA: Free Press, 2003.

42 Chambers DA, Glasgow RE, Stange KC. The dynamic sustainability framework: addressing the paradox of sustainment amid ongoing change. Implement Sci $2013 ; 8: 117$. 